\title{
Consideraciones acerca del uso de la variable etnia/raza en investigación epidemiológica para la Salud Pública: A propósito de investigaciones en inequidades
}

\author{
Jairo Vanegas $L^{1}$, Marcelo Villalón $C^{2}$, Carlos Valenzuela $Y^{3}$. \\ Ethnicity and race as variables in \\ epidemiological research about inequity
}

Epidemiology analyzes differences in states of health and disease of populations. Public Policies are established considering inequities associated with ethnicity and race. In this context, the identification of vulnerable groups for concentration of resources is relevant. Nevertheless, the lack of a clear definition of these variables might lead to biased results and interpretations. Two problems about the use of these variables are discussed. First, lack of a measurable and objective characteristic, even considering self reference (gold standard), considering that the opinion of a person can change in time. The second problem is a consequence of the former, basing research on a poorly defined variable. Uses of ethnicity and race variables between 1920-1999 in the American Journal of Epidemiology, Health Services Research and American Journal of Public Health were reviewed. In 919 articles, 27 different names identified to describe these variables and more than half did not describe the reason to use these variables. Almost half did not describe analytical methods. Although some articles found statistically significant relationships, less than half discussed those results. It has been suggested that there is enough evidence to exclude these variables in biomedical investigations. However, others propose that they cannot be excluded, given their multidimensional condition that includes social, cultural and genetic features. Therefore, provided the lack of clear definition, the assessment of ethnicity and race effects must be done as rigorously as possible (Rev Méd Chile 2008; 136: 637-44).

(Key words: Continental population groups; Epidemiology; Ethnology)

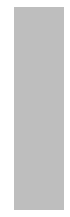

Recibido el 9 de mayo, 2006. Aceptado el 23 de julio, 2007.

${ }^{1}$ Programa Doctorado Salud Pública, Escuela Salud Pública, Facultad de Medicina, Universidad de Chile. Becario proyecto MECESUP UCH 0219. División de Promoción, Escuela Salud Pública, Facultad de Medicina, Universidad de Chile. ${ }^{3}$ Programa de Genética Humana, Instituto de Ciencias Biomédicas (ICBM), Facultad de Medicina, Universidad de Chile.

Correspondencia a: Jairo Vanegas L. Fax: 7377121. Teléfo-

no: 9786546. E mail: jvanegas@med.uchile.cl 
$\mathrm{E}^{\mathrm{n}}$ el ámbito de las inequidades en salud, la epidemiología analiza diferencias en el estado de salud-enfermedad de las poblaciones y las asocia con frecuencia a la variable raza/etnia. En este contexto se hace relevante identificar grupos vulnerables para focalización de recursos.

Investigaciones actuales sostienen que la variable raza no tendría base biológica, considerándola una falacia como concepto, y la variable etnia una representación inexacta de grupos genéticos inferidos $^{1,2}$. Más aún, se sugiere que la clasificación racial/étnica desde la genética podría no ser útil en estudios biomédicos, dado que refleja un número bastante pequeño de genes ${ }^{3,4}$. Por tanto, si no hay base biológica y es un mito la relevancia de las diferencias encontradas, el peso de éstas dentro de los modelos de causalidad disminuiría.

Sin embargo, las raciaciones y deraciaciones son procesos biológicos reales que ocurren en todas las especies, y que corresponden a un aislamiento reproductivo incompleto.

En Chile, por ejemplo, la población actual proviene de un proceso de raciación y deraciación conocido: (a) población mongoloide, separada de los caucásicos hace 45.000 años, (b) mongoloides que atravesaron el estrecho de Bering y poblaron América (30.000 a 40.000 años atrás), (c) que con el tiempo constituyeron la población amerindia. Finalmente, (d) durante el periodo de conquista (siglos XVI al XIX), en un proceso de mezcla entre amerindios y caucásicos (mayoritariamente españoles), se constituyó la población chilena actual ${ }^{5}$.

Más aún, en Chile el proceso se vincula estrechamente a una determinada estratificación socioeconómica: españoles de estrato social más alto, que no se mezclaron; la mezcla entre españoles europeos y mujeres mestizas en el estrato medio; finalmente, el estrato más bajo concentró las mezclas entre mestizos-mestizos o mestizos-amerindios. Recíprocos apareamientos entre mujeres europeas y hombres amerindios rara vez ocurrieron.

Tanto la variable etnia/raza así como la conformación de estratos socioeconómicos, son aspectos a considerar en los análisis de las inequidades en salud. Los autores abordan algunas consideraciones de la imprecisión conceptual de esta variable, su tratamiento e implicaciones metodológicas para la investigación en salud pública y las políticas.

\section{DESARROLL}

Estudios recientes se han enfocado en diferencias raciales/étnicas, particularmente entre negros y blancos, categorías que se asocian con indicadores socio-económicos ${ }^{6,7}$.

Consideremos la siguiente afirmación: "La principal diferencia existente entre pueblos se basa en su cultura y no en su raza/etnia"8. Por ejemplo, en Estados Unidos de Norteamérica las personas de raza negra habitualmente son asociadas con clases sociales pobres, cuando la población blanca es mayoritaria en el grupo de pobres y no todos en la población negra son pobres.

Por otro lado, las personas de raza negra son una representación de la integración génica mundial igual que los blancos. Un aborigen originario de Australia, un zulú de África del Sur y un kiuyu de Kenya son todos de raza negra, aunque su origen difiera geográficamente. De hecho, la variabilidad del ámbito social, cultural y genético de la variable raza/etnia aún no logra ser explicada del todo ${ }^{9}$.

Al principio del siglo XIX el concepto de raza dividió las poblaciones basándose en características físicas, postura que declinó al final de la segunda guerra mundial. Actualmente, raza/ etnia es utilizada más bien enfatizando el origen socioeconómico ${ }^{11,12}$.

La variable raza/etnia usada con propósito clasificatorio es susceptible a los cambios en los ámbitos políticos-sociales o migratorios producidos a través del tiempo. En Estados Unidos de Norteamérica en 1790, una persona de piel negra era catalogada como esclava, en 1850 como negra o mulata, en 1890 de ascendencia negra, en 1950 como negra y en 2000 como negra, afro-americana. Pero hoy, una persona si decidiera "autorreferirse" (autorreferencia considerada el estándar de oro en la clasificación de raza/etnia), podría clasificase blanca o indio americana 0 nativa de Alaska ${ }^{13}$.

Lo anterior evidenciaría a la variable raza/etnia como constructo social rigiendo distribución de riesgos y oportunidades en una sociedad, donde la pertenencia étnica refleja un acervo cultural y la raza mide una exposición de identidad implicando una imposición social asociada a dichas categonías ${ }^{8}$.

En las ciencias sociales existen tres corrientes de pensamiento acerca de la variable raza/etnia. 
La esencialista que la considera una cualidad primordial, dada de manera natural desde que nace el individuo; la situacional, en que la pertenencia es cuestión de actitud, percepción y sentimientos, por lo tanto, en la medida que éstas varían cambia también su pertenencia de origen; $\mathrm{y}$ por último, la corriente de atributos históricos y simbólicos, un colectivo cultural, mitos, recuerdos, religión, costumbres, lengua o instituciones propias que definirían raza/ etnia ${ }^{14,15}$.

Sin embargo, la perspectiva biológica agrega una historia genealógica, de ancestría o relaciones de parentesco, no consideradas en las corrientes descritas $^{16}$ (Figura 1).

En el mismo sentido, la expresión étnica e indígena se usan erróneamente como sinónimos. Etnia se refiere a un colectivo que se identifica a sí mismo y por otros en función de ciertos elementos comunes, como idioma, religión, tribus, características físicas. Indígena, en cambio, hace referencia a una persona que desciende de los pueblos que habitaban tierras nativas antes de la llegada de los conquistadores (caso de Chile y América) y que luego quedaron incorporadas dentro de los límites territoriales de los países actuales ${ }^{17,18}$.

Esta imprecisión conceptual repercute en las mediciones y resultados de los estudios ${ }^{19}$. Dada la multidimensionalidad y su interacción con diversos determinantes sociales, su uso como factor causal pudiera ser considerado débil, de no tener un tratamiento metodológico adecuado. Igualmente se produce confusión habitual entre raza y estrato socioeconómico o sociocultural, pues se confunde cluster social con cluster racial. De hecho, en estos estudios no se hace mención a diferencias genómicas y fenotípicas importantes y estadísticamente significativas, por ejemplo el color de piel, incidencia de anemia falciforme, grupo Rh y otras ${ }^{16}$.

En las definiciones antes revisadas se evita sistemáticamente hacer mención a la pertenencia reproductiva, rasgo central del concepto biológico de raza. Raza es un proceso biológico de partición reproductiva inexorable y no un constructo social, debido a que las poblaciones no son panmícticas. Es decir, no todos los individuos tienen la misma probabilidad de aparearse entre sí, ni el apareamiento es al azar. Se trata de un proceso dado al interior de las especies, que permite clasificar en subespecies, las razas, que son comunidades reproductivas parcialmente aisladas ${ }^{16}$.

Con lo anterior no se pretende que lo biológico sea el único referente, pero no debe desconocerse como elemento central en la discusión de la definición raza/etnia, tanto por motivos conceptuales como operacionales, que permitirían precisar de qué se habla en cada caso ${ }^{10}$.

En Chile ha sido propuesto un Marco de Referencia Sociogenético para estudios en Salud Pública. El fundamento de dicho marco es la demostración que el porcentaje de mezcla aborigen es casi inexistente en estratos socioeconómicos altos, y surge e incrementa en una gradiente hasta llegar a ser casi el $50 \%$ en los estratos bajos. Los estratos sociogenéticos son: alto (5\%), de población sin componente amerindio, un estrato medio (20\%) con $20 \%$ de mezcla amerindia, y un estrato bajo (75\%) con $35 \%$ a $40 \%$ de mezcla amerindia. Las implicancias de las diferencias sociogenéticas condicionarían las estructuras antropométricas, de inmunidad, morbilidad y mortalidad, que sumadas a las diferencias ambientales y socioculturales conllevan a desarrollar inequidades sociales ${ }^{10}$.

$$
\text { Raza }\left\{\begin{array}{l}
\cdot \text { Cultural. Elementos de identidad y tradición comunes } \\
\cdot \text { Social. Prácticas sociales comunes } \\
\cdot \text { Genética. Ancestría }
\end{array}\right.
$$

*Elaboración propia.

Figura 1. Multidimensionalidad de raza. 
El desafío por tanto, consiste en cómo medir la variable raza/etnia, pues al analizar estudios que emplean la variable raza/etnia se identifican al menos dos problemas. Primero, la falta de una característica mensurable y objetiva, aun tomando en cuenta la autorreferencia (considerado el "estándar de oro" para la clasificación), dado que la opinión de una persona puede variar en el tiempo.

En Estados Unidos de Norteamérica, la Oficina Management and Budget clasifica la población tomando en cuenta cinco grupos raciales, blancos, negros 0 afroamericanos, indio americanos 0 nativos de Alaska, asiáticos y nativos de Hawai/ Islas del Pacífico y además una categoría étnica, de hispánicos y no hispánicos. Sin embargo, estas categorías son heterogéneas y aunque utilizadas en investigación, su valor es limitado ${ }^{20}$.

En el caso de Latinoamérica y el Caribe, de quince países que realizaron censos en 2000, trece incorporaron al menos una pregunta para identificar personas como parte de una etnia (ante la recomendación de Naciones Unidas para identificar grupos étnicos). Algunas de las preguntas se basaron en autorreferencia, otras en lengua materna y otras en el idioma que hablaba la persona. Como era de esperar, la magnitud de la población indígena varió según los diversos criterios dificultando comparaciones entre países ${ }^{21}$.

El segundo problema, consecuencia del primero, es la ambigüedad esperable de los resultados de investigaciones que no han precisado su variable de estudio. Centrar las investigaciones sobre una variable sin una definición precisa (conceptual y operacional), atribuye la disparidad en salud a las diferencias raciales o étnicas de forma equívoca ${ }^{22}$.

Algunos autores, Ahdieh L (1996), Hahn RA (1996), Jones CP (1991) y Williams DR (1994), sugieren pautas metodológicas al usar raza/etnia como variable de investigación ${ }^{23-26}$. Entre las principales recomendaciones se mencionan diferenciar la variable, justificar su uso, describir el método empleado y ser cautos en las comparaciones o generalización de resultados, dada la variabilidad del concepto.

Una revisión sistemática de artículos que usaron la variable raza/etnia entre 1920 y 1999 en revistas como American Journal Epidemiology, Health Services Research y American Journal of
Public Health, identifican 27 nombres diferentes para describir el concepto raza/etnia en 919 artículos. Se describen las categorías negros hispánicos y negros no hispánicos, blancos/otros y negros y otros, y ni uno ni otros, como negros no blancos, no minoritarios (otros) y otros no blancos. El 57,4\% no describió el propósito del uso de la variable y en $48,7 \%$ no se explicaron los métodos de análisis. El 55,3\% de los artículos encontró relación estadísticamente significativa para raza/etnia, pero solamente $30,4 \%$ de los autores discutieron estos resultados ${ }^{27}$.

Por otra parte, la variable raza/etnia puede actuar como un factor de confusión o de interacción. Dicha situación puede llevar a interpretar un efecto no real o exagerar una asociación (confusión positiva) o por el contrario, atenuar la asociación e incluso producir una inversión de la asociación real (confusión negativa) ${ }^{28}$.

Modelos contrafactuales de causalidad definen la causa en relación a un efecto, por tanto, los factores considerados causas pueden manipularse plausiblemente, lo cual es imposible para la raza dada su característica fija ${ }^{16}$. Investigadores sugieren que un verdadero efecto causal de la raza se verificaría como un contraste en la distribución de los efectos en individuos manipulados en varios estados raciales/étnicos, como por ejemplo, la probabilidad $(\mathrm{Y}=\mathrm{y} \mid$ conjunto [raza $=$ negros] $)$ vs probabilidad $\left(\mathrm{Y}=\mathrm{y} \mid\right.$ conjunto $[\mathrm{raza}=\text { blancos] })^{30}$. En este caso sí sería aceptable una distribución contrafactual, dado el efecto de la distribución para blancos siendo hipotéticamente negros. Este tipo de análisis resulta absurdo para la variable raza/ etnia ${ }^{31,16}$.

Siendo una característica fija, los individuos en una sociedad no podrían libremente adoptar una identidad racial/étnica deseada, a diferencia de factores como dieta, status socioeconómico 0 características vecinales, que sí podrían tener una distribución diferenciada. Igualmente, algunas variables involucradas en los análisis podrían ser eslabones intermedios de un modelo causal. Por tanto, ajustar por raza/etnia más las otras variables, provocaría distorsión en las asociaciones $^{32,33}$.

Las variables independientes relacionadas con el entorno étnico y situación socioeconómica, incluidas en un modelo de regresión sin tomar en cuenta su posible correlación entre sí con la 
exposición plantean un problema de colinealidad, generando coeficientes de regresión difíciles de interpretar. Por tanto, realizar ajustes innecesarios llevaría a distorsiones de la medida de asociación, sobreestimando o subestimando los resultados ${ }^{34-36}$.

Por otro lado, los estudios demográficos establecen la distribución espacial poblacional desde el punto de vista socioeconómico, racial/étnico, migraciones, etc., identificando factores que expliquen estas distribuciones y cómo localidades cercanas geográficamente se influyen entre sí en la adopción de prácticas sociales. Se plantea así un problema metodológico de autocorrelación que afecta las estimaciones espaciales ${ }^{36}$.

La autocorrelación espacial se presenta cuando el valor que alcanza una variable en una observación determinada depende de los valores obtenidos en otras observaciones, lo que ocurre con las unidades espaciales que tienden a relacionarse entre sí, en especial las más próximas. Si la variable asume valores similares a unidades geográficas cercanas, esto da lugar a los llamados clusters. (Por ejemplo, áreas urbanas pobres tienden a estar cerca de otras áreas pobres). Esto violaría el supuesto de independencia de las observaciones generando problemas en las estimaciones de modelos de regresión lineal de mínimos cuadrados.

Por otro lado, la autocorrelación mostraría la influencia recíproca entre unidades de observación que pudieran ser producto de fuerzas sociales, políticas o económicas que tienden a agrupar a las poblaciones con rasgos comunes ${ }^{37}$. En el caso de la raza se trata de clusters poblacionales unidos por ancestría y reproducción compartida, y por lo tanto no es posible no esperar autocorrelación ${ }^{16}$.

Un aspecto metodológico más. Los procesos etiológicos extemos a los individuos definen mejor los contrastes causales, por ejemplo los estudios experimentales para investigar discriminación. Los efectos causales de raza/ etnia directamente estimados por el contraste de los efectos de distribución en cada tratamiento, justifica su uso como exposición válida ${ }^{30}$.

Dos ejemplos a considerar:

El estudio "The effect of race and sex on physicians' recommendations for cardiac catheterization" evaluó, a través de un diseño experimental controlado, las recomendaciones terapéuticas de los médicos a pacientes con distintos tipos de dolor torácico. La hipótesis afirmaba que la raza y el sexo de los pacientes influían las recomendacio- nes médicas con respecto al cateterismo cardíaco. El estudio concluye que efectivamente esta variable condicionaba la decisión del médico evidenciando un sesgo de prejuicio, que ocurre cuando la pertenencia del paciente a un grupo determinado activa automáticamente un estereotipo en la memoria del médico más allá del grado de prejuicio que el profesional tenga ${ }^{38}$.

En "Association of race/ethnicity with emergency department wait time Pediatrics", la hipótesis fue determinar si los tiempos de espera para niños menores de 15 años, tratados en las unidades de emergencia en los Estados Unidos de Norteamérica, se asociaban con la raza/ etnia de los pacientes. En una muestra representativa de 15.920 niños que acudieron a unidades de emergencia se encontró diferencias estadísticamente significativas entre los grupos. Para niños de raza blanca la espera fue de $54,5 \pm 0,1 \mathrm{~min}$, comparados con $48,7 \pm 0,5$ min $(p<0,01)$ y de $38,5 \pm 1,6$ min para niños de raza negra e hispanos blancos ( $p<0,02)$. El modelo de regresión lineal ajustado por variables género, raza/etnia, estatus socioeconómico, tipo de seguro, hospital y clasificación triage, sugirió que la raza/etnia fue factor determinante en los tiempos de espera $(p<0,01)^{39,40}$.

Algunas variables podrían considerarse eslabones intermedios dentro de modelos causales y al ser incluidos en un modelo de regresión conllevaría a sobreajuste. Habría que agregar que no fueron contemplados factores relevantes como la disminución de los servicios de atención primaria y el hecho que algunos padres no hablan inglés, lo cual contribuye a saturar las unidades de emergencia, aumentando tiempos de espera y mala clasificación de los pacientes por problemas de comunicación ${ }^{41-43}$.

\section{ConClusiones}

Es importante llamar la atención acerca de la variable raza/ etnia, ampliamente usada, pero cuya definición aún es imprecisa. Su consideración como constructo social plantea un manejo complejo, dada su relación con múltiples factores determinantes que no pueden ser reducibles.

El aumento del interés por incluir esta variable en los estudios refleja la dinámica de los cambios demográficos, la diversidad de grupos originarios 
o racialmente diferentes, categorías que se vinculan a indicadores socioeconómicos, obligando a cambios constantes en los conceptos y forma de clasificación poblacional, dificultando comparaciones entre países.

La variable etnia/raza pareciera tener más usos potenciales como determinante social que como determinante biológico de la salud. Sin embargo, está demostrado que la estructura de morbilidad genómica es dependiente de la raza (como realidad sociocultural y genética), y por lo tanto ppor qué separar lo sociocultural de lo genético? (Figura 1). Podría ser un error intentar desagregar lo no desagregable, pues la raza/etnia es una condición socio-cultural-genética y no es posible separarla conceptualmente sin que esto no lleve a consecuencias graves.

Los efectos de la agrupación racial deben ser estimados desde el apareamiento, por lo que tratar de estimar el efecto de las razas por la atención de salud o educación es un error conceptual grave, pues estos procesos se dan posteriormente. La conformación de la raza es un proceso en el tiempo cuyo hito crítico es la etapa de reproducción, por lo que pretender atribuir causalidad a la raza respecto de sistemas sociales resultaría improcedente.

De la misma manera, la falta de precisión conceptual y los métodos que fuerzan una relación causal, pueden repercutir en los resultados.
Desde el punto de vista metodológico sobresalen dificultades por el no cumplimiento de supuestos en algunos modelos estadísticos, debido a la complejidad e íntima relación entre covariantes, lo que conlleva a la subestimación o sobreestimación de resultados. Desde una perspectiva operacional se ha encontrado una parapropuesta para Chile que pudiera enriquecer y precisar el estudio de la variable raza/etnia y su uso para el estudio de inequidad en salud como es el Marco del Gradiente Sociogenético (Figura 2).

Finalmente, dejamos planteadas algunas preguntas:

- ¿Cómo se pueden focalizar recursos en población de mayor riesgo como los grupos originarios o racialmente diferentes, con herramientas de insuficiente precisión?

- ¿Es posible construir herramientas más precisas que las actuales? ¿Cómo?

- En las encuestas y censos poblacionales de América Latina se pregunta por la variable raza/etnia, sin considerar la multidimensionalidad de esta variable, ¿es posible concluir adecuadamente a partir de tales encuestas?

- En Chile, zes adecuado el instrumento y las preguntas de autorreferencia usadas actualmente? En aquellos estudios que dicen establecer asociaciones en un sentido $u$ otro, ise conoce el proceso por el que se ha definido la variable raza/etnia?

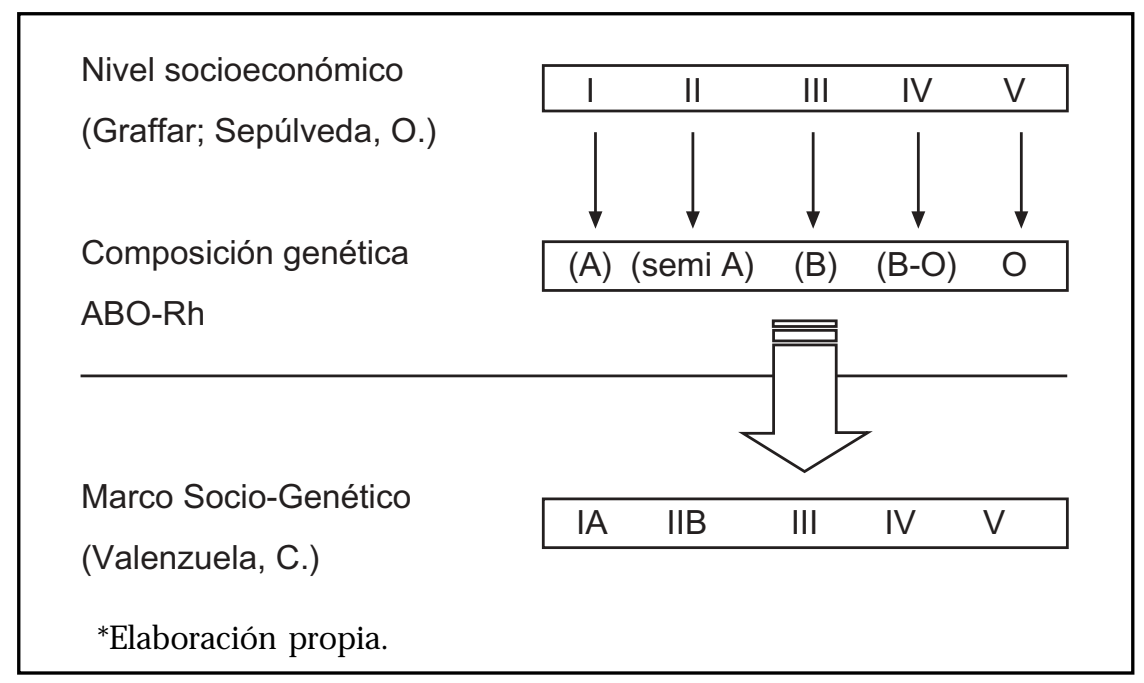

Figura 2. Asociación entre clasificación de estratos socioeconómicos y grupos ABO como bases para la construcción del marco sociogenético. 


\section{REFERENCIAS}

1. Schwartz RS. Perfil racial en investigaciones médicas. N Engl J Med 2001; 344: 1392-3.

2. Wilson JF, Weale ME, Smith AC, Gratrix F, Fletcher B, Thомas MF eт aL. Estructura genética en la población de la variable de la respuesta a las drogas. Nature Genetic 2001; 29: 265-9.

3. GonZÁLEZ E. The importance of RACE and Ethnic Background in Biomedical Research and Clinical Practice. New England of Medicine 2003; 348; 1170-5.

4. AfShari R, Bhopal R. Changing pattern of use of ethnicity and race in scientific literature. International Journal of Epidemiology 2002; 31: 1074-6.

5. ValenZuela CY, HarB Z. Socioeconomic associative mating in Santiago, Chile: a demonstration using stochastic matrices of mother-child relationships applied to ABO groups. Soc Biol 1977; 24: 225-33.

6. Kufman J, Cooper R, McGee L. Socioeconomic Status and Health in Black and Whites: The problem of residual confounding and the resiliency of race. Epidemiology 1997; 8: 621-8.

7. WINKER M. Measuring race and ethnicity: why and how? JAMA 2004; 292: 1612-4.

8. Jones C. Invite Commentary: RACE, Racism, and the practice of epidemiology. Am J Epidemiology 2001; 154: 299-304.

9. Cavau-Sforza LL, Menozzi P, Piazza A. The History and geography of human genes. Princeton, NJ: Princeton University Press, 1994; 19-20.

10. Valenzueia C. Marco de Referencia Sociogenético para los Estudios de Salud Pública en Chile. Revista Chilena de Pediatría 1984; Vol 55: 123-7.

11. Sentor P, Bhopal RS. Ethnicity as a variable in epidemiological research. BMJ 1994; 309: 327-9.

12. Bhopal R. Is research into ethnicity and health racist, unsound, or important science? BMJ 1997; 314: 1751-6.

13. Ignatiev N. How the Irish became white. New York, NY: Routledge, 1995.

14. Sмттн A. La identidad nacional, Trama Editorial, 1997, Madrid.

15. Belo A, Rangel M. Etnicidad, raza y equidad en América Latina y el Caribe. Comisión Económica para América Latina y el Caribe. CEPAL 2000. LC/ R. 1967/rev.1.

16. VALENZUela C. Genetic Epidemiology of Diabetes in Chile. 1998. INTA, Universidad de Chile.
17. Stavenhagen R. Los conflictos étnicos y sus repercusiones en la sociedad internacional. Revista internacional de ciencias sociales. 1991. № 157, Organización de las Naciones Unidas para la Educación, la Ciencia y la Cultura (UNESCO).

18. Hompenhayn M, Bewo A. Discriminación étnicoracial y xenofobia en América Latina y el Caribe. CEPAL, 2001, Santiago, Chile.

19. BHopal R. Glossary of terms relating to ethnicity and race: for reflection and diabate. J Epidemiol Community Health 2004; 58; 441-5.

20. Office of Management and Budget. Standards for maintaining, collecting and presenting federal data on race and ethnicity. Federal Register 62 FR 58781-58790, 1997. Disponible en: http:// www.whitehouse.gov/omb/fedreg/ombdir15.html (Accessed January 19, 2004).

21. Comisión Económica para América Latina y el Caribe. Pueblos Indígenas y Afrodescendientes de América Latina y el Caribe: Información sociodemográfica para políticas y programas. CEPAL, 2005. Santiago de Chile.

22. JONES CP. Levels of racism: a theoretic framework and a gardener's tale. Am J Public Health 2000; 90: 1212-5.

23. AHIDIEH L, HAHN RA. Use of the terms "race", "ethnicity" and "national origins": a review of article in the American Journal of Public Health, 1980-1989. Ethn Health 1996; 1: 95-8.

24. HaHn RA, Stroup DA. Race and ethnicity in public health surveillance: criteria for the scientific use of social categories. Public Health Rep 1994; 109: 7-15.

25. Jones CP, La Veist TA, Lume-Blantom M. "RACE" in the epidemiologic literature: an examination of the American Journal of Epidemiology, 19211900. Am J Epidemiol 1991; 134: 1079-84.

26. Wiwams DR. The concept of race in Health Services Research: 1966 to 1990. Health Serv Res 1994; 29: 261-74.

27. Dawn R, CASTILO E, Kinsay S. Four year review of the use of RACE and ethnicity in epidemiologic and public health research. Am J Epidemiol 2004; 159: 611-9.

28. Irala J, Martínez A, Guilén F. ¿Qué es una variable de confusión? Méd Clin (Barc) 2001; 117: 377-85.

29. Greenland S, Robins JM, Pearl J. Confounding and Collpsibility in Causal Inference. Stat Sci 1999; 14: 29-47. 
30. Pearl J. Causality: models, reasoning and inference. Cambridge, United Kingdom: Cambridge University Press, 2000.

31. MuntaneR C. Invited Commentary: social mechanisms, race, and social epidemiology. Am J Epidemiol 1999; 150: 121-6.

32. LiNk BG, Phelan J. Social conditions as fundamental causes of disease. J Health So Behav 1995; 38: 80-94.

33. Robiins AS, Whittemore AS, Thom DH. Differences in socio-economic status and survival among White and Black men with prostate cancer. Am J Epidemiol 2000; 151; 409-16.

34. Kaufman J, Cooper R. Commentary: Considerations for Use of Racial/Ethnic Classification in Etiologic Research. Am J Epidemiol 2001; 154: 291-8.

35. Jokin I, Estévez J, Martínez M, Seguia M. Epidemiología Aplicada. En: Errores en la estimación de medidas de asociación en estudios epidemiológicos. España: Editorial Ariel, 2004; 287-90.

36. Szкцо M, Nieto J. Epidemiología Intermedia, Conceptos y Aplicaciones. En: Estratificación y ajustes, Análisis Multivariantes en Epidemiología. España: Editorial Díaz de Santos S.A. Año 2003; pág 288.
37. Voss P, Logg D, Hammer R, Friedman S. "County Child Poverty Rates in the US: A Spatial Regression Approach". 2006; 25: 369-91.

38. Kevin a Schulman, BerLin JA, Hariess W, Kerner J, Sistrunk S, Gersh B et al. The effect of race and sex on physicians' recommendations for cardiac catheterisation. N Engl JMed 1999; 340: 618-26.

39. Catherine J. Association of race/ethnicity with Emergency Department Wait Time. Pediatrics 2005; 115; 310-5.

40. Kressin NR, Chang B, Hendricks A, Kazis LE. Agreement between administrative data and patients self-reports of rac/ethnicity. Am J Public Health 2003; 93: 1734-9.

41. Boehmer U, Kressin NR, BerLowitz DR, Christianse C, Kazis L, Jones J. Self-reported vs administrative race/ethnicity data and study results. Am J Public Health 2002; 92: 1471-3.

42. Coniglaro J, Whittle J, Good CB, Hanusa BH, Passman LJ, Lofgren RP et al. Understanding racial, variation in the use of coronary revascularization procedures: the role of clinical factors. Arch Intern Med 2000; 160; 1329-35.

43. http://atlasgeneticsoncology.org/Educ/Hardy Sp.htm (Accessed February, 2004). 